\title{
Conditioning vs. Excitation Time for Estimating Impedance Parameters of the Human Arm
}

\author{
Dominic Lakatos, Florian Petit and Patrick van der Smagt
}

\begin{abstract}
The human arm's capability to alter its impedance has motivated multiple developments of robotic manipulators and control methods. It provides advantages during manipulation such as robustness against external disturbances and task adaptability. However, how the impedance of the arm is set depends on the manipulation situation; a general procedure is lacking. This paper aims to fill this gap by providing a method to estimate the impedance parameters of the human arm, while taking the numerical stability of the approach into account. A dynamic arm model and an identification method is presented. Confidential criteria to determine the accuracy of the estimated parameters are given. Finally, the procedure is validated in an experiment with a human subject and the results are discussed.
\end{abstract}

\section{INTRODUCTION}

Dynamic interaction with the environment means handling impacts and unknown contact forces. Therefore compliant systems are active topics of research in the field of humanoid and service robotics. The DLR light-weight robot LWR-III [1] is an example for active impedance controlled robots, i.e. the joint torques are adjusted to behave like a springdamper system, and the DLR Hand-Arm System [2], [3] is actuated via passive variable stiffness joints. Surpassing traditional rigid robots, the control loops of both systems are extended with additional impedance parameter, viz. stiffness and damping.

Even though the above-mentioned systems are outstanding examples of variable impedance robots, one important issue is not resolved: how are the impedance parameters set to optimally perform a predefined task? Traditionally, robotic tasks are only defined in target end-effector positions or, in some cases, end-effector trajectories; but the impedance around these positions or trajectories remains a matter of common sense, at best. For instance, when performing a peg-in-hole task, high stiffness in the perpendicular and low stiffness in the lateral directions, so as to allow for imprecise positioning while solving the task, appears to be useful. But how do we find general rules-of-thumb for setting these extra parameters?

Beside heuristic methods tuning the impedance parameters, mimicking the behavior of the human arm is an auspicious field of research, and leads to what we call biologically inspired robotics. By measuring and subsequently analyzing human arm impedance parameters, we can attempt to extract general rules and project these to the robotic domain.

The authors are with the Institute of Robotics and Mechatronics, German Aerospace Center (DLR), D-82234 Oberpfaffenhofen, Germany \{ dominic.lakatos, florian.petit, smagt\}@dlr.de
However, analyzing the mechanics of the human arm needs a reliable method for measuring the impedance parameters.

\section{A. Related work}

In [4], [5], [6], [7], [8], [9], [10], [11], several methods to analyze and measure the impedance of the human arm are proposed. The underlaying model in those works describes the inverse dynamic in the transversal plane by a two link, two joint multi body system, where viscoelasticities acting on the hinges.

The most straightforward method is described in [7]. Here the endpoint of the arm is perturbed by a planar manipulator. After reaching a steady-state in position, the ratio of the difference in force (after and before perturbation) and displacement is considered to estimate the stiffness. Since the performance of the manipulator is limited, the steady-state is not reached before $500 \mathrm{~ms}$ after onset of the perturbation. An extension to the method proposed by [7] is described in [10]. It is used to identify the complete dynamic model, i.e., inertia, stiffness and damping. Therefore the endpoint of the arm is disturbed by a smooth displacement. In order to estimate the model parameters, a two times integrated second order linear model is fitted to the observed motion and force data. The length of the observation window is about $450 \mathrm{~ms}$. Another approach to identify the whole dynamic model is based on frequency analysis, and was developed in [8], [9]. This non-parametric identification method uses a bandlimited white noise in position, to excite the human arm. By means of the fast Fourier transforms of the recorded force and displacement data, scalar transfer functions were computed. Obtaining the impedance parameters, these transfer functions can be parametrized by nonlinear optimization. To meet the requirements for this identification method, the length of the observation window must be about $30 \mathrm{~s}$ [9].

The mentioned methods are useful to measure impedance parameters in steady arm postures. Some approaches to analyze the mechanics during arm movement are given in [4], [5], [6]. The method developed in [4] is based on the prediction of the unperturbed motion trajectory. Stiffness can be estimated by comparing position and force of the perturbed and unperturbed trajectories. Therefore the perturbed motion must be formed to have a plateau phase, during which the error velocity (between perturbed and unperturbed motion) is zero. The stiffness was estimated in the interval of $120 \mathrm{~ms}$ and $180 \mathrm{~ms}$. The method is restricted to estimating the stiffness, i.e., velocity-proportional damping forces were not acting under those conditions. Accurate estimates can only be obtained when Coriolis/centrifugal forces are taken 


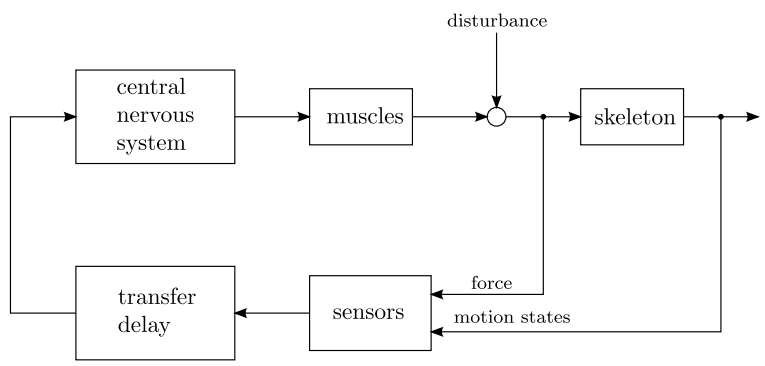

Fig. 1. Control loop of the neuromusculoskeletal system.

into account, but that means that inertia parameters must be known. This drawback can be overcome by calculus of variation of the nonlinear equations of the arm model, as it was done before in [5], [6]. The resulting model describes the dynamic of the arm between free and disturbed trajectories, and is linear in the unknown parameters (i.e., inertia, stiffness and damping). Hence local linearized impedance can be estimated at a working point during movement by means of force perturbations. The recorded data used to identify the parameters is observed in an interval beginning at the onset of the perturbation with a length of $280 \mathrm{~ms}$.

\section{B. This work}

Since the neuromusculoskeletal system of the human arm is closed-loop controlled via the central nervous system (CNS), at least the stiffness parameters vary over time (see Figure 1 and [12]). That means, disturbances are detected by the sensors, and the delayed transmitted signals will cause a change of muscle activation and mechanical properties (e.g., stiffness and damping). This functional chain is called reflex in biological context. The most important reflex loops, affecting the impedance measurements, will be described as follows:

- The stretch reflex is activated by stretching the muscle fibers. This so-called low-latency reflex occurs after $25 \mathrm{~ms}$ to $50 \mathrm{~ms}$ and is controlled through the spine; the parameters of this type of feedback are fixed.

- The spinal reflex acts on joint level, e.g., flexing the forearm (due to activation of $\mathrm{m}$. biceps) activates the m. triceps in order to restore the equilibrium of joint torques. The latency of spinal reflexes is $70 \mathrm{~ms}$ to $110 \mathrm{~ms}$. This type of feedback is controlled through the spine, and its parameters are fixed.

- Long-latency reflexes with reaction time of at least $110 \mathrm{~ms}$ are operating at human cognitive level, after which time we can deliberately steer our muscles; the feedback parameters are cognitively determined and cannot be realistically modelled.

As described in the above-mentioned publications, identifying the parameters of the whole dynamic arm model (i.e., inertia, stiffness and damping) requires observing the data in an interval of at least $280 \mathrm{~ms}$ length (after onset of perturbation). Since the reaction time of reflexes is in the range of $25 \mathrm{~ms}$ to $110 \mathrm{~ms}$, this is in conflict with the assumption that stiffness and damping parameters are constant; especially the long-latency reflexes are to be avoided. The topic of this paper is minimizing the length of the observation window under a viewpoint of conditioning (for linear optimization) and criteria derived from basic mechanical laws (e.g. integrability of vector fields). This will be done by proposing a model and identification method, defining criteria for reliability of the parameter estimate, and demonstrating, by experiments, which interval length is sufficient.

\section{Modeling OF THE NEUROMUSCULOSKELETAL SYSTEM}

The neuromusculoskeletal system of the human arm can be modeled as a rigid multi-body system, composed of two bodies (i.e., the upper arm and the forearm). The muscles are considered as force elements, actuating the bodies in joint space, and coupled via motion (and neural) inputs. Additionally it is assumed that the muscles mass can be added to the mass of skeletal bodies.

\section{A. Rigid body dynamics of the skeletal system}

The inverse dynamic equations of the masses for $n$ joint variables $\mathbf{q} \in \mathbb{R}^{n}$ is given by

$$
\boldsymbol{\Gamma}(\mathbf{q}, \dot{\mathbf{q}}, \ddot{\mathbf{q}}, \boldsymbol{\xi})=\mathbf{M}(\mathbf{q}, \boldsymbol{\xi}) \ddot{\mathbf{q}}+\mathbf{C}(\mathbf{q}, \dot{\mathbf{q}}, \boldsymbol{\xi}) \dot{\mathbf{q}}+\mathbf{g}(\mathbf{q}, \boldsymbol{\xi}),
$$

where $\mathbf{M} \in \mathbb{R}^{n \times n}$ is the positive definite and symmetric mass matrix, $\mathbf{C} \in \mathbb{R}^{n \times n}$ the Coriolis/centrifugal matrix ( $\mathbf{C}$ can be computed to make $\dot{\mathbf{M}}-2 \mathbf{C}$ skew symmetric), and $\mathbf{g} \in$ $\mathbb{R}^{n}$ the vector of gravity forces. $\boldsymbol{\xi}$ is the vector of the constant so-called base inertial parameters, determining the mass, mass moment of inertia, and first-order mass moments of the bodies. It is necessary that the components $\xi_{i}$ are linearly independent (i.e., $\forall \alpha_{i} \neq 0, \alpha_{1} \xi_{1}+\alpha_{2} \xi_{2}+\ldots+\alpha_{k} \xi_{k} \neq 0$, where $k$ is the length of $\boldsymbol{\xi}$ ) in order to be identifiable [13].

\section{B. Impedance of the neuromuscular system}

The model of a muscle is given by the general state-spacerepresentation

$$
\begin{aligned}
& \dot{\mathbf{x}}=f(\mathbf{x}, \mathbf{u}) \\
& \mathbf{y}=g(\mathbf{x})
\end{aligned}
$$

where $f(\mathbf{x}, \mathbf{u})$ describes the dynamic of the muscle states $\mathbf{x}, \mathbf{u}$ is the input vector, and $g(\mathbf{x})$ the output function. An explicit description of $f(\mathbf{x}, \mathbf{u})$ is beyond the scope of this paper ${ }^{1}$. Assuming $f(\mathbf{x}, \mathbf{u})$ can be integrated, one can write an explicit function for the forces generated by a muscle

$$
h_{m}=h_{m}\left(l_{m}, l_{0}, v_{m}, a\right)
$$

where $l_{m}$ is the muscle length, $l_{0}$ the initial length, $v_{m}$ the muscle shortening rate, and $a$ the neural activation. Collecting the force functions and input variables of each muscle in vectors, the impedance of the arm can be written in the form

$$
\mathbf{h}(\mathbf{q}, \dot{\mathbf{q}}, \mathbf{a})=\mathbf{L} \mathbf{h}_{m}(\mathbf{q}, \dot{\mathbf{q}}, \mathbf{a}), \quad \text { where } \quad \mathbf{h} \in \mathbb{R}^{n} .
$$

\footnotetext{
${ }^{1}$ possible descriptions can be found in, e.g., [14], [15]
} 
Herein the matrix of moment arms $\mathbf{L}$ is introduced, and the following terms were substituted:

$$
\begin{aligned}
\Delta \mathbf{l}_{m} & =-\mathbf{L}^{T} \Delta \mathbf{q} \\
\Delta \mathbf{v}_{m} & =-\mathbf{L}^{T} \dot{\mathbf{q}}
\end{aligned}
$$

where $\Delta \mathbf{l}_{m}=\mathbf{l}_{m}-\mathbf{l}_{0}$ and $\Delta \mathbf{q}=\mathbf{q}-\mathbf{q}_{0}$. Notice $\mathbf{q}_{0}$ is assumed to be constant; its dependency in (4) is neglected accordingly.

\section{Partial linearized model}

Superimposing the torques due to muscle impedance (4) on the inverse dynamic (1) leads to the equations of motion

$$
\mathbf{M}(\mathbf{q}, \boldsymbol{\xi}) \ddot{\mathbf{q}}+\mathbf{C}(\mathbf{q}, \dot{\mathbf{q}}, \boldsymbol{\xi}) \dot{\mathbf{q}}+\mathbf{g}(\mathbf{q}, \boldsymbol{\xi})+\mathbf{h}(\mathbf{q}, \dot{\mathbf{q}}, \mathbf{a})=\tau_{\text {ext }},
$$

where $\boldsymbol{\tau}_{\text {ext }}$ are externally applied forces/torques. In order to describe the impedance part in $(6), \mathbf{h}(\mathbf{q}, \dot{\mathbf{q}}, \mathbf{a})$ can be linearized around an operating point. Hence the Taylor approximation in $\mathbf{q}_{0} \equiv \mathbf{q}\left(t_{0}\right), \dot{\mathbf{q}}_{0}=0$ and $\mathbf{a}_{0} \equiv \mathbf{a}\left(t_{0}\right)$ (neglecting higher order terms) holds:

$$
\begin{aligned}
\mathbf{h}^{\star}= & \left.\mathbf{h}\right|_{\mathbf{q}_{0}, \mathbf{a}_{0}}+\left.\frac{\partial \mathbf{h}(\mathbf{q}, \dot{\mathbf{q}}, \mathbf{a})}{\partial \mathbf{q}}\right|_{\mathbf{q}_{0}, \mathbf{a}_{0}} \Delta \mathbf{q} \\
& +\left.\frac{\partial \mathbf{h}(\mathbf{q}, \dot{\mathbf{q}}, \mathbf{a})}{\partial \dot{\mathbf{q}}}\right|_{\mathbf{q}_{0}, \mathbf{a}_{0}} \Delta \dot{\mathbf{q}}+\left.\frac{\partial \mathbf{h}(\mathbf{q}, \dot{\mathbf{q}}, \mathbf{a})}{\partial \mathbf{a}}\right|_{\mathbf{q}_{0}, \mathbf{a}_{0}} \Delta \mathbf{a}
\end{aligned}
$$

Since $\mathbf{q}_{0}$ is an equilibrium point and assuming $\mathbf{a}(t)=$ const. (this is only valid during latency time, i.e., no reflexes acting) (7) reduces to

$$
\mathbf{h}^{\star}=\mathbf{K} \Delta \mathbf{q}+\mathbf{D} \dot{\mathbf{q}}
$$

where the constant Jacobian's $\mathbf{K} \in \mathbb{R}^{n \times n}$ and $\mathbf{D} \in \mathbb{R}^{n \times n}$ represent the positive definite, symmetric matrices of local linearized stiffness and damping.

Substituting (8) in (6) leads to the partial linearized model of the neuromusculoskeletal system, which will be used for further investigations.

\section{IDENTIFICATION METHODS}

First, the identification model, which is linear in the parameters, is derived. Second, trajectories exciting the identification model suitably are proposed. Finally, some confidential criteria for evaluating the "quality" of the parameter estimates are proposed.

\section{A. Identification model}

In the following, the multi-joint arm movement in the transversal plane will be considered. Therefore the gravity term in (6) vanishes, and the dynamic model can be written as

$$
\underbrace{\mathbf{M}(\mathbf{q}, \boldsymbol{\xi}) \ddot{\mathbf{q}}+(\mathbf{C}(\mathbf{q}, \dot{\mathbf{q}}, \boldsymbol{\xi})+\mathbf{D}) \dot{\mathbf{q}}+\mathbf{K} \Delta \mathbf{q}}_{\Psi(\ddot{\mathbf{q}}, \dot{\mathbf{q}}, \Delta \mathbf{q}, \boldsymbol{\xi}, \mathbf{D}, \mathbf{K},)}=\Delta \tau_{\mathrm{ext}}
$$

where $\mathbf{q} \in \mathbb{R}^{2}$, and $\boldsymbol{\Psi}$ is the vector of inverse dynamics (including impedance). For the planar model with two degrees of freedom ${ }^{2}$ it can be shown that $\boldsymbol{\xi} \in \mathbb{R}^{3}$. Thus the unknown model parameters are

$$
\boldsymbol{\zeta}=\left[\xi_{1}, \xi_{2}, \xi_{3}, D_{11}, D_{12}, D_{21}, D_{22}, K_{11}, K_{12}, K_{21}, K_{22}\right]^{T},
$$

and the identification model can be written as

$$
\mathbf{W} \zeta=\mathbf{y},
$$

where $\mathbf{W} \in \mathbb{R}^{r \times c}$ is the observation matrix and $\mathbf{y} \in \mathbb{R}^{r}$ the output vector ( $r$ is the number of scalar linear equations and $c$ the number of unknown parameters). If the observation interval consists of $N$ samples, $\mathbf{W}$ and $\mathbf{y}$ are composed of

$$
\mathbf{W}=\left[\begin{array}{c}
\mathbf{X}(1) \\
\mathbf{X}(2) \\
\vdots \\
\mathbf{X}(N)
\end{array}\right], \quad \mathbf{y}=\left[\begin{array}{c}
\Delta \boldsymbol{\tau}_{\text {ext }}(1) \\
\Delta \boldsymbol{\tau}_{\text {ext }}(2) \\
\vdots \\
\Delta \boldsymbol{\tau}_{\text {ext }}(N)
\end{array}\right]
$$

In the case of the planar arm model, the matrix of independent variables is given by the Jacobian

$$
\begin{aligned}
\mathbf{X} & =\left(\frac{\partial \mathbf{\Psi}(\ddot{\mathbf{q}}, \dot{\mathbf{q}}, \Delta \mathbf{q}, \boldsymbol{\xi}, \mathbf{D}, \mathbf{K},)}{\partial \boldsymbol{\zeta}}\right) \in \mathbb{R}^{2 \times 11} \\
X_{1,1} & =\ddot{q}_{1} \\
X_{1,2} & =\left(2 \ddot{q}_{1}+\ddot{q}_{2}\right) \cos q_{2}-\left(2 \dot{q}_{1} \dot{q}_{2}+\dot{q}_{2}^{2}\right) \sin q_{2} \\
X_{1,3} & =\ddot{q}_{2} \\
X_{2,2} & =\ddot{q}_{1} \cos q_{2}+\dot{q}_{1}^{2} \sin q_{2} \\
X_{2,3} & =\ddot{q}_{1}+\ddot{q}_{2} \\
X_{1,4} & =X_{2,6}=\dot{q}_{1} \\
X_{1,5} & =X_{2,7}=\dot{q}_{2} \\
X_{1,8} & =X_{2,10}=\Delta q_{1} \\
X_{1,9} & =X_{2,11}=\Delta q_{2} \\
X_{1,6} & =X_{1,7}=X_{1,10}=X_{1,11}=X_{2,1} \\
& =X_{2,4}=X_{2,5}=X_{2,8}=X_{2,9}=0
\end{aligned}
$$

The least squares solution of the overdetermined system of linear equations (11) can be computed by

$$
\hat{\boldsymbol{\zeta}}=\left(\mathbf{W}^{T} \mathbf{W}\right)^{-1} \mathbf{W}^{T} \mathbf{y}=\mathbf{H}^{-1} \mathbf{W}^{T} \mathbf{y},
$$

where the Hessian $\mathbf{H}=\mathbf{W}^{T} \mathbf{W}$ is the second derivative of the squared error function.

\section{B. Excitation trajectory}

The "quality" of the parameter estimate (14) strongly depends on the choice of the excitation trajectory. As one can see from (13) the columns of $\mathbf{W}$ consists of displacements, velocities and accelerations terms. Thus, if the order of magnitudes of motion variables (e.g., between displacements and accelerations) are widely distributed, the conditioning of the Hessian diverges (i.e., $\mathbf{H}$ gets close to singular), that means the parameter estimate becomes inaccurate since $\mathbf{H}$ can no longer be stably inverted. A possible trajectory generation method is based on the minimum jerk theory, proposed by [16]. The solution of

$$
\min _{q(t)} \frac{1}{2} \int_{t=0}^{0.5} \dddot{q}(t)^{2} d t
$$

\footnotetext{
${ }^{2}$ E.g., consisting of the shoulder and elbow joints.
} 
is a fifth-order polynomial

$$
q(t)=a_{0}+a_{1} t+a_{2} t^{2}+a_{3} t^{3}+a_{4} t^{4}+a_{5} t^{5},
$$

see, e.g., [17]. The polynomial order of five is necessary to specify all boundary conditions for a point-to-point motion with equilibrium start/goal-points. In order to compare the ability of fifth-order polynomials compared to higher-order polynomials w.r.t. well-conditioned Hessians $\mathbf{H}$, the ratio

$$
C_{i j}=\frac{\frac{1}{t_{f}} \int_{t=0}^{t_{f}} \stackrel{i}{q}(t)^{2} d t}{\frac{1}{t_{f}} \int_{t=0}^{t_{f}} \stackrel{j}{q}(t)^{2} d t},
$$

is defined. Herein, $i / j$ denotes the $i$ th $/ j$ th time-derivative $t$, and $t_{f}$ the time elapsed for reaching the goal-point $q_{f}$. Notice, that the numerator/denominator are squared mean values of the motion variable $\stackrel{i / j}{q}(t)$. Thus, if $C_{i j}=1$ the order of magnitude between $\stackrel{i}{q}$ and $\stackrel{j}{q}$ is equal. Comparing a fifth- and sixth-order polynomial, the following boundary conditions are defined: $q(0)=0, q\left(t_{f}\right)=q_{f}, \dot{q}(0)=0$, $\dot{q}\left(t_{f}\right)=0, \ddot{q}(0)=0, \ddot{q}\left(t_{f}\right)=0$, and $\dddot{q}\left(t_{f}\right)=0$. The cross combinations of $C_{i j}$ (for $i=1,2$ and $j=2,3$, where $i<j$ ) of both polynomials are faced in Tab. I. For each

TABLE I

CONDITIONING RATIO COMPARING POLYNOMIALS

\begin{tabular}{c|ccc} 
order & $C_{12}$ & $C_{13}$ & $C_{23}$ \\
\hline 5th & $\frac{181}{660} t_{f}^{2}$ & $\frac{181}{7920} t_{f}^{4}$ & $\frac{1}{12} t_{f}^{2}$ \\
6th & $\frac{329}{1560} t_{f}^{2}$ & $\frac{329}{22880} t_{f}^{4}$ & $\frac{3}{44} t_{f}^{2}$
\end{tabular}

combination, the values $C_{i j}$ of the fifth-order polynomial are closer to 1 than the values of the sixth-order polynomial. Hence the profile of the fifth-order polynomial (16) is used to generate excitation trajectories.

\section{Confidential criteria}

Since the measurement procedure is biased, and the mathematical model is only an approximation of the real human arm behavior, confidential criteria are needed to evaluate the "quality" of the parameter estimate.

A measure quantifying the conditioning of a linear optimization problem, can be derived from the singular-value decomposition of the observation matrix $\mathbf{W}$ (see, e.g., [18])

$$
\mathbf{W}=\mathbf{U} \boldsymbol{\Sigma} \mathbf{V}^{T} \text {. }
$$

$\mathbf{U} \in \mathbb{R}^{r \times r}$ and $\mathbf{V} \in \mathbb{R}^{c \times c}$ are orthogonal matrices, and $\boldsymbol{\Sigma} \in \mathbb{R}^{r \times c}$ is diagonal, containing the singular values $\sigma_{i}$ in decreasing order. Thus the condition number is defined by

$$
\operatorname{cond}(\mathbf{W})=\frac{\sigma_{\max }}{\sigma_{\min }} .
$$

For $\operatorname{cond}(\mathbf{W})=1$ the problem is perfectly conditioned, and for $\operatorname{cond}(\mathbf{W}) \rightarrow \infty$ the Hessian $\mathbf{H}$ is singular. The condition number is a measure, evaluating the sensibility of the least squares solution due to model uncertainties. Since the model accuracy is unknown, it is hard to determine an upper limit for the condition number. Thus further criteria have to be addressed.

Two other confidential criteria used are based upon the stiffness matrix $\mathbf{K}$. The stiffness matrix of a captive mechanical systems is positive definite. Hence the general property

$$
\forall \delta \mathbf{q} \neq 0, \delta \mathbf{q}^{T} \mathbf{K} \delta \mathbf{q}>0
$$

holds ( $\delta \mathbf{q}$ is arbitrary). This can be interpreted as the strain energy, which must be positive for finite displacements. An extension to this property is derived from the force field

$$
\Delta \boldsymbol{\tau}(\Delta \mathbf{q})=\mathbf{K} \Delta \mathbf{q}
$$

which is a potential field for passive mechanical systems, if the integrability conditions

$$
\operatorname{curl}(\Delta \boldsymbol{\tau})=0 \Leftrightarrow\left(\mathbf{K}-\mathbf{K}^{T}\right)=0
$$

are fulfilled. Accordingly, the stiffness matrix $\mathbf{K}$ must be symmetric.

Summarizing the confidential criteria, one can trust the parameter estimate if

- the condition number (19) is small;

- $\mathbf{K}$ is positive definite (20);

- $\mathbf{K}$ is symmetric (22).

\section{EXPERIMENTAL RESULTS}

As stated above, the length of the observation interval should be less than the latency time of the CNS. This could be in conflict with the conditioning of the least squares identification procedure. In order to find a minimum observation interval empirically, which fulfills the proposed confidential criteria, an experiment will be performed.

\section{A. Experimental setup}

The experimental setup is shown in Fig. 2. The main part is a position/torque-controlled light-weight robot, which is used to perturb the human arm and measure the arm position via the joint sensors (i.e., manipulators forward kinematics). The participant is fixed to a body-contoured seat to allow only motions of the subject's arm. The robot and the seat are mounted on a metal frame standing on the ground.

A six-axis JR3 force/torque sensor is mounted on the robot's end effector, in order to measure the forces and torques applied by the human arm. The robot end effector is connected to the human arm via a plastic cuff, which includes a metal beam supporting the arm against gravity. Both force/torque and position data are recorded by the realtime operating system VxWORKS at the sampling rate of $1 \mathrm{kHz}$. 


\section{B. Methods}

One healthy subject was tested. The upper body was restrained by the shoulder belts in the seat, and the right forearm was fixed in the plastic cuff. The subject's arm was held in the initial configuration $\mathbf{q}_{0}=(0.08,1.00)$ by the robot. On a display the interaction forces the human arm is exerting on the robot's end effector were shown. The subject was instructed to exert a force of $5 \mathrm{~N}$ distal with least effort as possible, so as to prevent co-contraction. After a random waiting time of $1 \ldots 3 \mathrm{~s}$, the robot performed a perturbation in one of six randomly chosen directions. In order to estimate one parameter set, this procedure was repeated until the data of 30 trials had been recorded. The desired perturbation trajectories were computed in joint coordinates of the human arm by means of the fifth order polynomial (16). The desired perturbation amplitudes $\Delta \mathbf{q}_{f}=\mathbf{q}_{f}-\mathbf{q}_{0}$ were: $(0.05,0),(-0.05,0),(0,0.05),(0,-0.05),(0.05,-0.05)$ and $(-0.05,0.05)$. The experimental boundary conditions had been changed by varying the length of the interpolation interval $T_{k}=40 \mathrm{~ms}+k \cdot 10 \mathrm{~ms}$, where $k=1,2, \ldots, 26$. A typical perturbation is shown in Fig. 3 .

\section{Data analysis}

In order to estimate the $k$ th parameter set (10), the data observed in the interval $T_{\text {obs }}=T_{k}+70 \mathrm{~ms}$ (see Fig. 3) over 29 trials (one trial is used to validate the prediction) is considered in (12) and (13), respectively. Therefore the joint angles $\mathbf{q}$ are computed via an analytical inverse kinematics, by means of the recorded end effector position. After phase free low-pass filtering, the derivatives $\dot{\mathbf{q}}$ and $\ddot{\mathbf{q}}$ are computed by numerical differentiation. The external applied joint torques are obtained by $\boldsymbol{\tau}_{\text {ext }}=\mathbf{J}(\mathbf{q})^{T} \mathbf{F}_{\text {ext }}$, where $\mathbf{F}_{\text {ext }}$ are the Cartesian forces (measured by the JR3 force/torque sensor) and $\mathbf{J}(\mathbf{q})=\partial f(\mathbf{q}) / \partial \mathbf{q}$ is the Jacobian for the human arms end effector position $\mathbf{x}=f(\mathbf{q})$ ( $f$ must be a bijection). $\Delta \mathbf{q}=\mathbf{q}(t)-\mathbf{q}_{0}$ and $\Delta \boldsymbol{\tau}_{\text {ext }}=\boldsymbol{\tau}_{\text {ext }}(t)-\boldsymbol{\tau}_{0}$

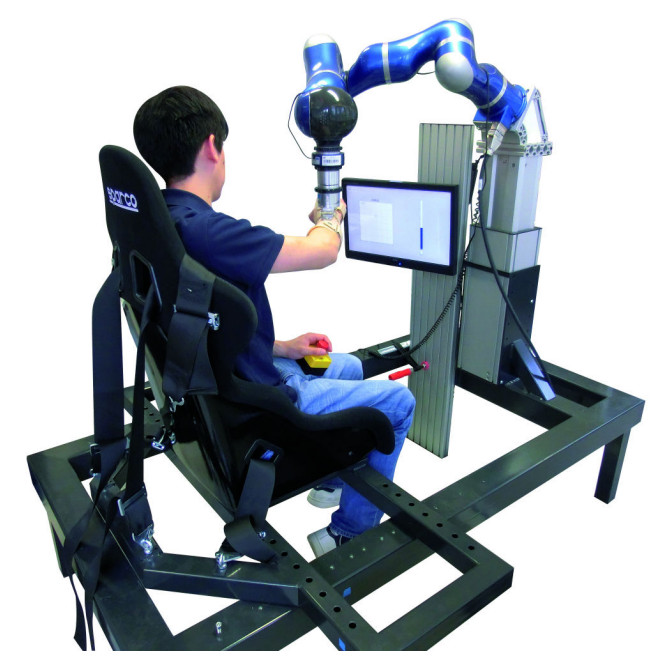

Fig. 2. Experimental setup

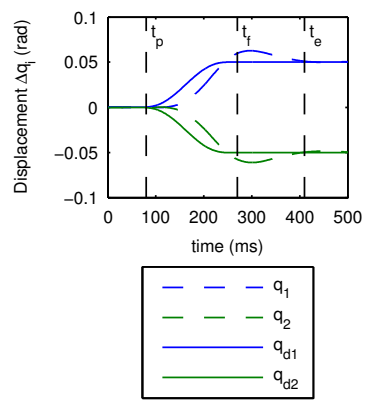

(a)

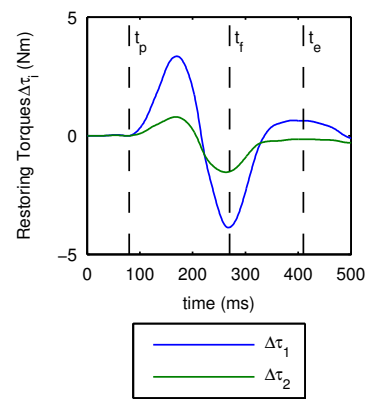

(b)
Fig. 3. Typical Perturbation: onset of perturbation is marked by $t_{p}$, interpolation interval length by $T_{k}=t_{f}-t_{p}$, and observation interval length by $T_{o b s}=t_{e}-t_{p}$.

are computed by the difference of the actual values at time $t$, and the mean values during the initial steady-state, e.g., $\mathbf{q}_{0}=\frac{1}{t_{p}} \int_{0}^{t_{p}} \mathbf{q}(t) d t$.

\section{Results}

The dependency between excitation time (i.e., length of observation interval $T_{\text {obs }}$ ) and confidential criteria (proposed in Sec. III-C) of the experimental parameter estimate is shown in Fig. 4. Evaluating whether the stiffness and damping matrix $\mathbf{K}$ and $\mathbf{D}$ are positive definite, the eigenvalues of the symmetric parts $\boldsymbol{\lambda}_{K}=\operatorname{eig}\left(\mathbf{K}_{s}\right)$ and $\boldsymbol{\lambda}_{D}=\operatorname{eig}\left(\mathbf{D}_{s}\right)$, where $\mathbf{K}_{s}=1 / 2\left(\mathbf{K}+\mathbf{K}^{T}\right)$ and $\mathbf{D}_{s}=1 / 2\left(\mathbf{D}+\mathbf{D}^{T}\right)$, are considered. Additionally, the components $K_{i j}$ and $D_{i j}$ are shown to assess the symmetries.

For $T_{\mathrm{obs}}>320 \mathrm{~ms}$ the stiffness matrix gets positive definite, and for $T_{\text {obs }}>370 \mathrm{~ms}$ stiffness and damping is quasi-symmetric. When $T_{\mathrm{obs}}>400 \mathrm{~ms}$, the whole parameter set is quasi-constant. Beside stiffness and damping values-which strongly depend on the experimental boundary conditions - the base inertial parameters $\boldsymbol{\xi}$ are comparable, and in the order of magnitude of values estimated in [5]. This motivates to analyze the recorded data with fixed $\boldsymbol{\xi}=(0.391,0.155,0.149)^{T}$, obtained from the full dynamic model identification (12). Thus the unknown model parameters are reduced to

$$
\boldsymbol{\zeta}_{\text {red }}=\left[D_{11}, D_{12}, D_{21}, D_{22}, K_{11}, K_{12}, K_{21}, K_{22}\right]^{T},
$$

which must be considered in the output vector

$$
\mathbf{y}_{i}=\Delta \boldsymbol{\tau}_{e x t}-(\mathbf{M}(\mathbf{q}, \boldsymbol{\xi}) \ddot{\mathbf{q}}+\mathbf{C}(\mathbf{q}, \dot{\mathbf{q}}, \boldsymbol{\xi}) \dot{\mathbf{q}}),
$$

and in the Jacobian of independent variables

$$
\mathbf{X}_{r e d}=\partial \mathbf{\Psi}(\dot{\mathbf{q}}, \Delta \mathbf{q}, \mathbf{D}, \mathbf{K}) / \partial \boldsymbol{\zeta}_{r e d}
$$

Substituting (23) to (25) in (11) and (12) respectively and using the same data and estimation procedure as above leads to the results shown in Fig. 5. Additionally, Fig. 6 depicts the prediction of $\Delta \boldsymbol{\tau}_{\text {ext }}$ for the data not used in the training set. 


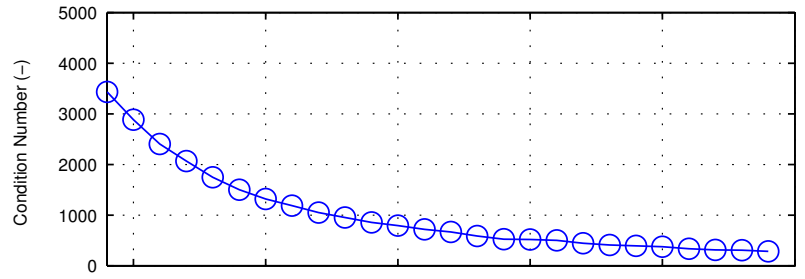

(a)

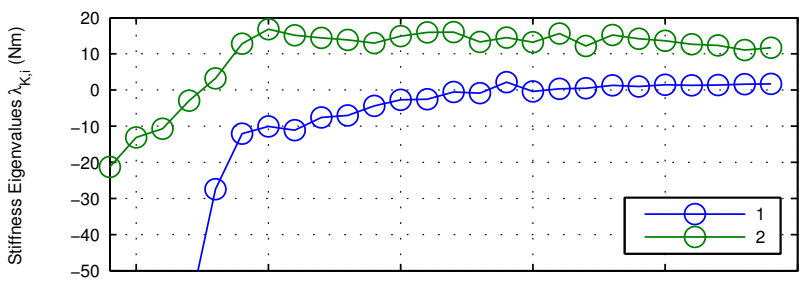

(b)

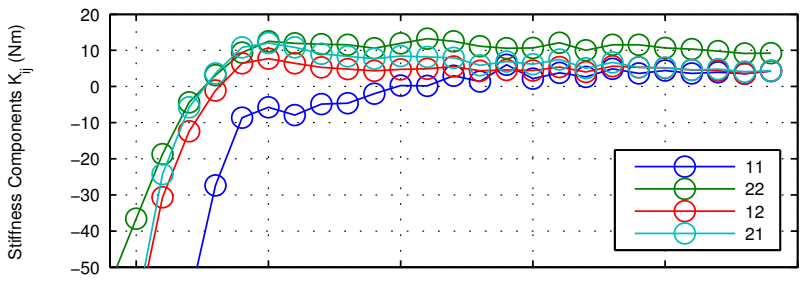

(c)

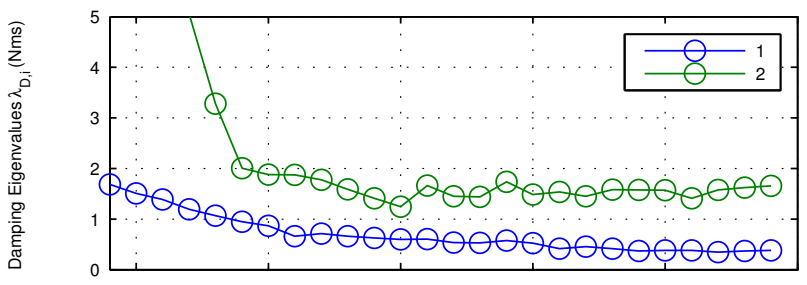

(d)

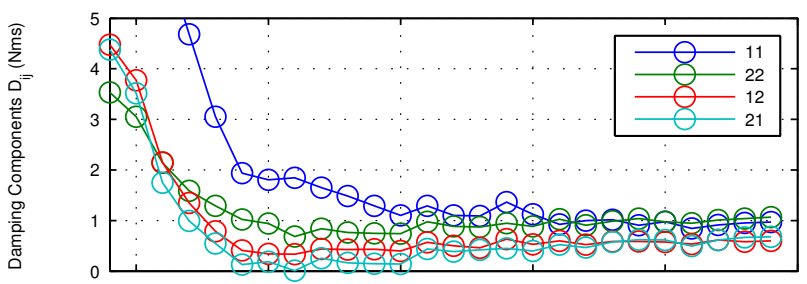

(e)

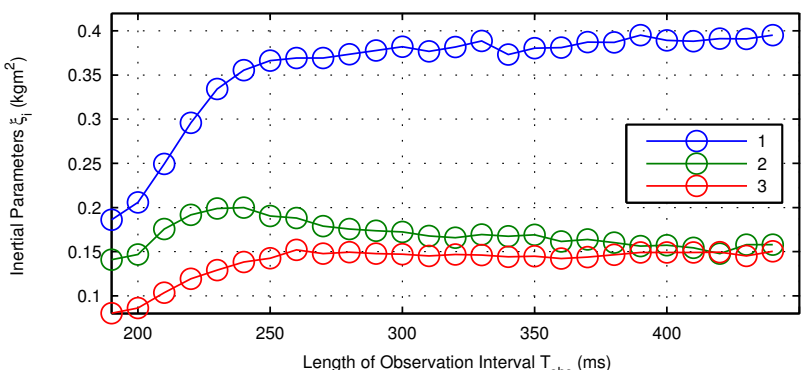

(f)

Fig. 4. Condition number, estimated stiffness, damping and inertial parameters over the length of the observation interval $T_{\text {obs }}$.

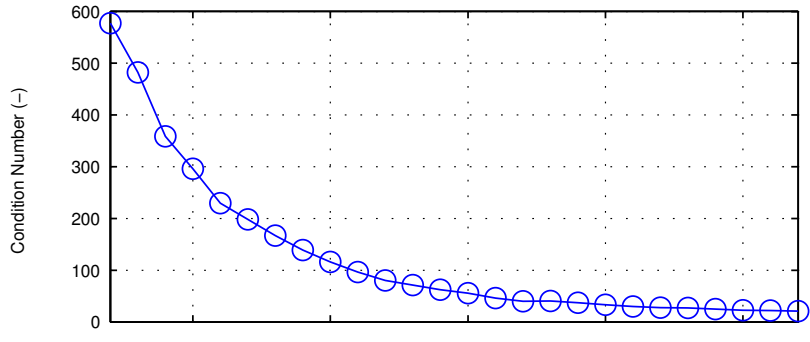

(a)

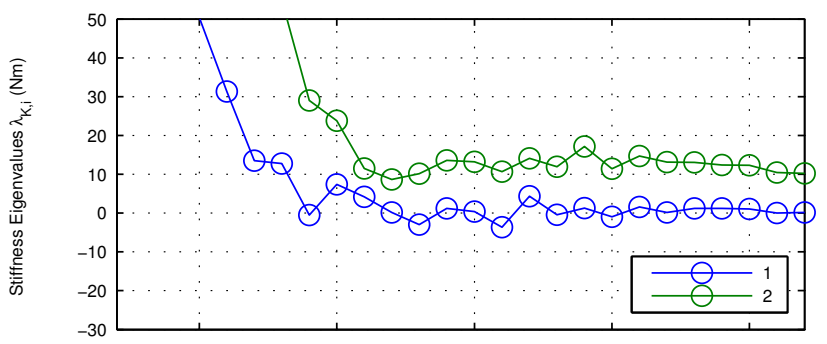

(b)

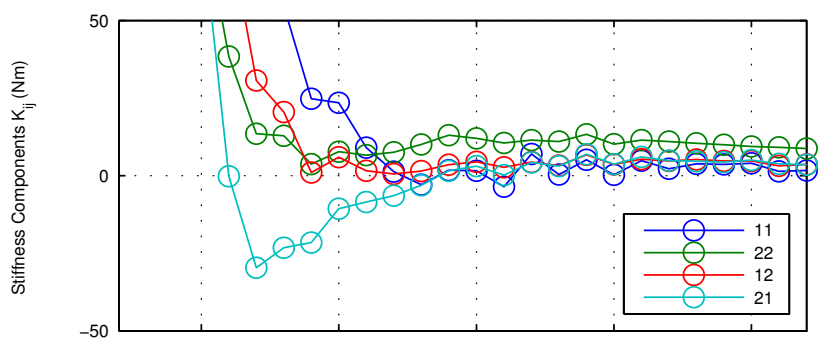

(c)

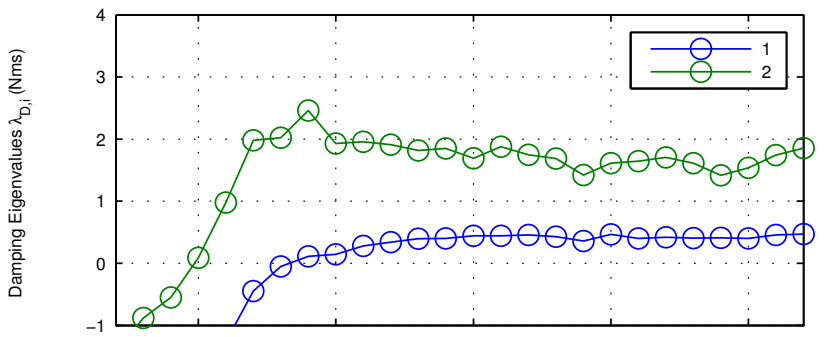

(d)

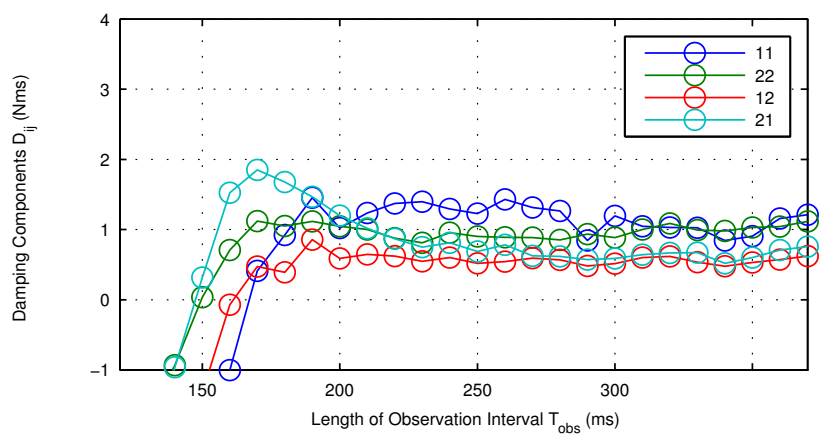

(e)

Fig. 5. Condition number, estimated stiffness and damping over the length of the observation interval $T_{\mathrm{obs}}$, where the inertial parameters are set to be constant and known. 


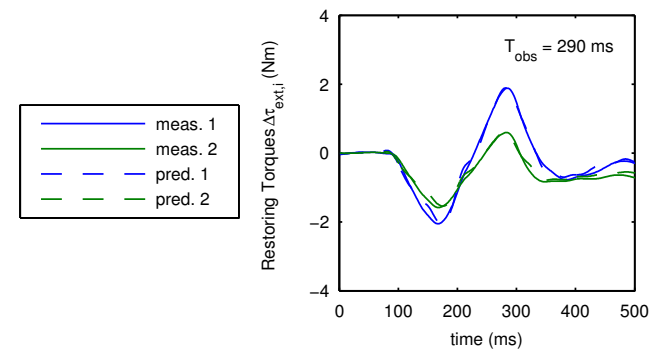

(a)

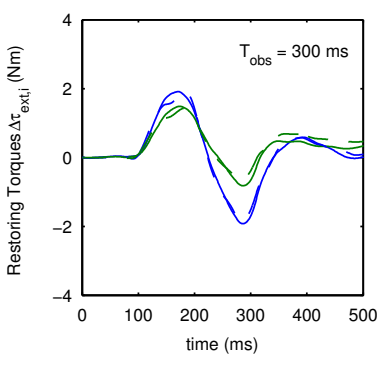

(b)

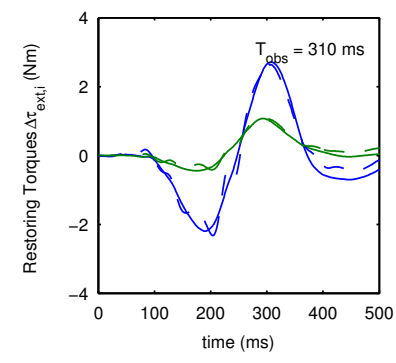

(c)
Fig. 6. Prediction of $\Delta \boldsymbol{\tau}_{\text {ext }}$ for the data not used in the training set.

\section{Conclusion}

In this paper a method for estimating the full dynamic model of the human arm is addressed, and confidential criteria evaluating the accuracy of the parameter estimate are proposed. The method is validated by an experiment. We have shown that accurately estimating the parameters of a partially linearized arm model (i.e., inertia, stiffness, damping) requires an observation window of about $370 \mathrm{~ms}$, which can be reduced to about $300 \mathrm{~ms}$ when the inertial parameters are known. This is an important result with a relevant impact on previous publications where human arm impedance parameters are measured. Conversely, the time window of about $300 \mathrm{~ms}$ is in clear contrast with the requirement of preventing cortical feedback or even spinal feedback in the measurements, since those feedback times are around $100 \mathrm{~ms}$.

With the method that we have introduced, the impedance parameters are obtained during a postural task. Due to the length of the observation window they can be seen as an average of "controlled" stiffness and damping. From a viewpoint of conditioning and spinal reflex time, an extension of this method to impedance estimation during arm motion may be prohibitive. We will therefore concentrate on measuring intrinsic limb impedance, i.e., the impedance of the tendonmusculoskeletal structure without controlled feedback. This would allow us to quantity the nonlinear relationship between, e.g., EMG, and impedance, and subsequently use that to estimate impedance parameters during arm motion. This in turn would allow us gain a deep insight in the interaction dynamics of the human arm and use subsequent models as guidelines for robotic impedance control.

\section{ACKNOWLEDGMENT}

This paper has been partly funded by the EC project STIFF, under Grant no. FP7-EC-231576.

\section{REFERENCES}

[1] A. Albu-Schäffer, S. Haddadin, C. Ott, A. Stemmer, T. Wimböck, and G. Hirzinger, "The DLR lightweight robot: design and control concepts for robots in human environments," Industrial Robot: An International Journal, vol. 34, pp. 376-385, 2007.

[2] M. Grebenstein and P. van der Smagt, "Antagonism for a highly anthropomorphic hand-arm system," Advanced Robotics, vol. 22, no. 1, pp. 39-55, 2008.

[3] M. Grebenstein, A. Albu-Schäffer, T. Bahls, M. Chalon, O. Eiberger, W. Friedl, R. Gruber, S. Haddadin, U. Hagn, R. Haslinger, H. Höppner, S. Jörg, M. Nickl, A. Nothhelfer, F. Petit, J. Reill, T. Wimböck, N. Seitz, S. Wolf, T. Wüsthoff, and G. Hirzinger, "The dlr hand arm system," in IEEE Conference on Robotics and Automation, 2011.

[4] E. Burdet, R. Osu, D. W. Franklin, T. Yoshioka, T. E. Milner, and M. Kawato, "A method for measuring endpoint stiffness during multijoint arm movements," Journal of Biomechanics, vol. 33, pp. 1705$1709,2000$.

[5] H. Gomi and M. Kawato, "Human arm stiffness and equilibrium-point trajectory during multi-joint movement," Biological Cybernetics, vol. 76, pp. 163-171, 1997, 10.1007/s004220050329. [Online]. Available: http://dx.doi.org/10.1007/s004220050329

[6] H. Gomi and R. Osu, "Task-dependent viscoelasticity of human multijoint arm and its spatial characteristics for interaction with environments," The Journal of Neuroscience, vol. 18, pp. 8965-8978, 1998.

[7] F. A. Mussa-Ivaldi, N. Hogan, and E. Bizzi, "Neural, mechanical, and geometric factors subserving arm posture in humans," The Journal of Neuroscience, vol. 5, pp. 2732-2743, 1985.

[8] E. J. Perreault, R. F. Kirsch, and A. M. Acosta, "Multiple-input, multiple-output system identification for characterization of limb stiffness dynamics," Biological Cybernetics, vol. 80, pp. 327-337, 1999.

[9] E. J. Perreault, R. F. Kirsch, and P. E. Crago, "Effects of voluntary force generation on the elastic components of endpoint stiffness," Experimental Brain Research, vol. 141, pp. 312-323, 2001.

[10] T. Tsuji, P. G. Morasso, K. Goto, and K. Ito, "Human hand impedance characteristics during maintained posture," Biological Cybernetics, vol. 72, pp. 475-485, 1995.

[11] N. Hogan, "The mechanics of multi-joint posture and movement control," Biological Cybernetics, vol. 52, pp. 315-331, 1985.

[12] H. van der Kooij, B. Koopman, and F. C. van der Helm, "Human motion control," 2008, reader for Delft University course.

[13] M. Gautier and W. Khalil, "Direct calculation of minimum set of inertial parameters of serial robots," in IEEE Transactions on Robotics and Automation, 1990.

[14] J. M. Winters and L. Stark, "Analysis of fundamental human movement patterns through the use of in-depth antagonistic muscle models," IEEE Transaction on Biomedical Engineering, vol. 32, pp. 826-839, 1985.

[15] —, "Muscle models: What is gained and what is lost by varying model complexity," Biological Cybernetics, vol. 55, pp. 403-420, 1987.

[16] T. Flash and N. Hogan, "The coordination of arm movements: an experimentally confirmed mathematical model," Journal of Neuroscience, vol. 5, pp. 1688-1703, 1985.

[17] R. Shadmehr and S. P. Wise, The computational neurobiology of reaching and pointing, R. Shadmehr and S. P. Wise, Eds. MIT Press, 2005.

[18] W. Khalil and E. Dombre, Modeling, Identification and Control of Robots. Hermes Penton Ltd, 2002. 